\title{
Journalistes et attachés de presse : regard anthropologique sur des professionnels « distingués »
}

\section{Alain Nivarlet}

\section{CpenEdition}

\section{Journals}

Édition électronique

URL : http://journals.openedition.org/communicationorganisation/1665

DOI : 10.4000/communicationorganisation. 1665

ISSN : 1775-3546

\section{Éditeur}

Presses universitaires de Bordeaux

\section{Édition imprimée}

Date de publication : 1 novembre 1993

ISSN : 1168-5549

\section{Référence électronique}

Alain Nivarlet, « Journalistes et attachés de presse : regard anthropologique sur des professionnels « distingués » », Communication et organisation [En ligne], 4 | 1993, mis en ligne le 26 mars 2012, consulté le 19 avril 2019. URL : http://journals.openedition.org/communicationorganisation/1665 DOI : 10.4000/communicationorganisation. 1665

Ce document a été généré automatiquement le 19 avril 2019

(c) Presses universitaires de Bordeaux 


\title{
Journalistes et attachés de presse : regard anthropologique sur des professionnels « distingués »
}

\author{
Alain Nivarlet
}

1 Le titre de cette réflexion assigne volontairement au terme «distingués » une signification ambivalente. Distingués, au sens commun, les journalistes et, plus encore, les attachés de presse, le sont certainement. Ils occupent également une place particulière au sein de l'univers de la communication professionnalisée; à ce titre ils mentent encore l'épithète de « séparés ». Au-delà de cette commune distinction, il se pourrait bien que ces communicateurs cherchent encore à se distinguer entre eux, les uns par rapport aux autres. Non simplement par la fonction, ce qui paraît presque évident, mais par des comportements moins manifestes qu'une étude anthropologique, par exemple, pourrait tenter de mettre au jour.

2 Je me propose donc de rendre compte d'observations de terrain récoltées lors de manifestations de relations publiques exclusivement destinées, on verra plus loin pourquoi, aux professionnels de la presse. C'est bien d'une approche anthropologique de processus déterminés de communication qu'il s'agit, dans le but avoué de débusquer la « distinction » là où elle surgit, révélée par une attitude, institué par un clivage, confortés par une tradition plus ou moins ritualisée. Ce faisant, je tenterai de faire échapper les relations publiques à la double instrumentalisation des pratiques, dans une littérature dominée par l'irritante question du «How to do ? ", et instrumentalisation des praticiens, dont la carte de visite dans les milieux des médias semble être le vieux label « La voix de son maître ».

3 Plus fondamentalement, fidèle à une démarche inductive, nous renverrons dos-à-dos, les deux grandes catégories de professionnels qui animent le champ de la communication, les chargés de relations publiques et les attachés de presse d'un côté, les journalistes de l'autre, mettant ainsi en évidence une des fractures invisibles qui polarisent cet univers en apparence si homogène. 


\section{« Communicateurs » et « informateurs » face-à-face}

4 Renouvelant à notre manière le clivage omniprésent dans les écoles de communication ${ }^{1}$, acceptons d'emblée le paradigme mis en évidence dans un ouvrage devenu classique : la communication n'est rien d'autre qu'une certaine quantité d'information prise au sein d'une relation intersubjective ${ }^{2}$.

5 Précisément, situé par notre démarche anthropologique en un lieu d'où cette dernière devient parfaitement visible, je procéderai à une «enquête sur une relation» (sur un " comportement »), convaincu que celle-ci fournira les clés des démarches qualifiées de « communicatives » d'une part et d'« informatives » d'autre part.

6 Cette dernière remarque pourrait d'ailleurs soulever une question légitime éternel retour d'Hydre - : comment distinguer les « relations publiques » des autres pratiques du champ professionnel de la communication? La chose est malaisée mais aussi, heureusement, relativement inutile pour nous. Les professionnels de la communication chargés des contacts avec la presse se définissent clairement comme des chargés de relations publiques, ce qui nous suffit amplement. Les pratiques adoptées en ces circonstances, en effet, revêtent un triple aspect : primo, les professionnels des R.P. font figure de «lieutenants ", de re-présentants, c'est-à-dire, comme le signalait récemment Nathalie Heinich, de personnes qui «suppléent à l'absence » de ceux qu'ils représentent ${ }^{3}$. Secundo, les manifestations organisées se caractérisent par une certaine ostentation, laquelle n'est ici qu'un autre nom de la représentation au sen théatral. Tertio, cette double représentation se déroule sur un horizon, un «background » aux contours imprécis mais prégnant, celui de l'image globale de l'entreprise représentée 4 .

7 Pour en terminer, avec ces préambules, insistons sur le point que notre réflexion n'est pas menée in abstracto, mais au contraire en toute fidélité à des observation, nombreuses et répétées, sur le terrain. Notre corpus est en fait constitué d'un ensemble de manifestations axées sur une présentation de services ou de produits à la presse par des R.P. professionnels, mandatés soit par la firme qui les emploie, soit par une agence spécialisée engagée par elle. Les rencontres en question sont des opérations où des moyens majeurs ont été mis en œuvre (déplacements à l'étranger, séjours, réceptions, conférences multimédias, etc.) et qui peuvent de ce fait être considérées comme des archétypes de ce qui se fait de mieux dans le secteur.

8 Je dois en outre à la vérité de dire que mon statut d'observateur dans ces circonstances était ambivalent. D'une part, les acteurs en présence ignoraient la plupart du temps qu'ils étaient l'objet d'une étude anthropologique. D'autre part, j'avais moi-même, à chaque fois un statut d'acteur à part entière, soit comme chargé de R.P., soit, le plus souvent, comme journaliste-correspondant du quotidien bruxellois Le Soir. Statut discutable s'il en est, dont la vertu principale était de me donner, comme on va le voir, une position tout à fait stratégique au sein du champ à observer.

9 Outre ces raisons d'opportunité, on peut invoquer quelques motifs déterminants de s'intéresser aux relations entre chargés de R.P. et journalistes. Les journalistes, avec l'univers des médias qu'ils représentent, ne constituent jamais, par rapport aux professionnels de la communication d'entreprise, que «l'autre » versant du secteur de la communication. Les journalistes sont, si l'on veut à nouveau faire référence au paradigme rappelé ci-dessus, les professionnels de l'information, c'est-à-dire de la communication en 
quelque sorte dépouillée de sa dimension relationnelle ${ }^{5}$. Cette articulation si évidente n'a pourtant guère fait l'objet d'investigations systématiques selon ce paradigme. L'essentiel des publications disponibles se trouve mentionné dans un article de Jean Charron, qui analyse lui-même le phénomène à partir d'une théorie psycho-sociologique de l'échange et de la négociation 6 .

Cette manière brutale de poser les choses, héritage de Watzlawick, indique assez notre point vue : « inviter la presse » lorsqu'on est chargé de R.P., c'est d'abord, stricto sensu, tenter d'insérer dans le jeu de la relation publique, du comportement qui séduit, ceux qui, pour eux-mêmes et aux yeux de l'opinion publique, se réclament avec force de la "pure information ".

11 Sur le plan pratique, cette tentative d'assimilation ne pose guère de problèmes aux professionnels des R.P. Rompus à cet exercice, ils recourent è la solution éprouvée consistant à offrir aux journalistes leur participation à une représentation (une mise en scène) dont l'information sera la très officielle raison d'être. Information-événement ou information-prétexte, peu importe, tant le registre du savoir-faire professionnel est étendu. Première étape de cette démarche de séduction : l'invitation qui offre déjà, à elle seule, un aperçu de l'étendue de ce registre du prétexte, du «mensonge » en train de s'insinuer à petites doses acceptables :

12 - «Au terme de cette conférence de presse, vous aurez l'occasion de poser les questions qui intéressent vos lecteurs et auditeurs durant le repas convivial qui sera servi au Château de... »

13 - «Ce Média Workshop se tiendra en prélude au 6e colloque international de à Florence. Chacun d'entre vous aura ainsi l'occasion, après le symposium-satellite de [nom de la firme invitante], de participer aux sessions plénières avec les meilleurs spécialistes mondiaux de... »

La troisième formulation retenue, dans son caractère lapidaire, résumé parfaitement l'interaction qui va se jouer : - «Au cours de ce repas, notre directeur, monsieur..., s'entretiendra personnellement avec vous et répondra à toutes vos questions ».

L'invitation est lancée, le ton est donné, la pièce peut commencer.

\section{La rencontre : les relations à l'œuvre}

\section{Convivialité et pragmatisme professionnel}

Dès l'instant du premier contact entre le chargé de R.P. et les journalistes «cibles " (le carton d'invitation), l'accent est donc mis sur deux aspects certes essentiels de la future rencontre, mais dont le rapprochement laisse rêveur : le contenu informatif d'une part, entendu comme "la seule chose qui peut vraiment mobiliser un journaliste $»^{7}$, et le contexte particulièrement heureux qui entourera la démarche informative. Lequel contexte, curieusement, occupe en moyenne plus de $80 \%$ du temps de l'activité considérée ${ }^{8}$.

La chose est d'autant plus étrange que, de l'avis général, « les journalistes n'ont jamais le temps ». L'objectif des chargés de R.P. et de leurs commanditaires est simple : faute de pouvoir acheter directement des «espaces rédactionnels (le filtre sélectif que constitue l'indépendance des journalistes les en empêche), ils achètent - ou, plus exactement, ils 
offrent - du temps pour leurs interlocuteurs, dans l'espoir que cette latitude temporelle favorisera un contact de qualité avec eux et se traduira ultérieurement, par des informations favorables dans les médias.

19 Cette curieuse répartition du temps de la rencontre publique semble imposer la sentence de Pierre Bourdieu: "La vérité de l'interaction "»; en l'occurrence, la vérité de la rencontre prévue est située ailleurs que dans l'information qui en est le prétexte avéré ou, autrement dit encore, la vérité communicationnelle (relationnelle, comportementale) ne saurait se lire que dans les comportements eux-mêmes.

$20 \mathrm{Au}$ fond, considérant l'interaction du point de vue d'un sociologue qui se méfiait de la communication, je serais tenté d'affirmer que la vérité de l'interaction qui rassemble journalistes et chargés de R.P au sein d'une rencontre de presse est à chercher du côté du cadre de celle-ci, étant entendu que ce cadre n'est rien d'autre qu'une structure sociale tenue pour acquise par les acteurs en présence et qui permet de mettre en évidence la signification réelle de l'échange ${ }^{10}$.

21 Suivant ce raisonnement, la figure sociale qui confère sa signification réelle à l'interaction étudiée est celle du " mensonge » professionnel. J'entends par la un mensonge qui n'est serait un qu'au sein d'un contexte déontologique beaucoup plus large (où les prétextes ne peuvent tenir lieu de textes, où la relation ne peut jamais infléchir l'information )mais que la nature purement professionnelle de l'échange désamorcerait en quelque sorte. Dans un contexte éthique général, on conçoit en effet aisément que séduction et information soient nettement distinguées; cependant, lorsque les acteurs sociaux agissent au nom d'une entreprise, les objectifs de celle-ci (profit, notoriété, image...) deviennent ipso facto les leurs, ce qui a pour effet de relativiser leur discours : « On sait bien qu'ils ne sont pas ici pour dire du mal de leur boite", se répètent entre eux les journalistes. Bref, les relations publiques ont leurs lois, qui ne sont pas toujours celles de la cité, et dont l'interaction livre le cadre. Le pragmatisme professionnel est de mise ; les régies communes de la vie sociale, fussent-elles d'ordre éthique, subissent un épochè légitime.

On ne saurait trop insister sur le fait que tous les acteurs observés dans ce cadre sont, dans leurs domaines respectif, des professionnels. Des spécialistes de la communication, tantôt formés par la pratique du métier (première génération des R.P. en Europe), tantôt formés dans les mêmes écoles de communication (pour la génération plus jeune). La portée de cette remarque est d'ailleurs tout à fait générale : les journalistes et les attachés de presse en présence, même s'ils ne sont pas des " pros » au sens strict (c'est-à-dire qui tireraient de leur pratique la majorité de leurs revenus et qui y emploieraient la majeure partie de leur temps), se doivent de feindre de l'être. C'est en effet une des caractéristiques frappantes de ce milieu que d'assimiler les «nouveaux », les « indépendants », les «free lance », les «stagiaires " ou les «jobistes » comme des acteurs à part entière, dûment mandatés par leurs médias ou agences, donc pleinement légitimes.

Tous ces "outsiders", précipités dans une situation dont ils ne maîtrisent pas toujours les mœurs et les codes spécifiques, s'astreignent à une discipline particulière pour ne pas dénoter par rapports a leurs confrères. Ainsi les jeunes journalistes éviteront de poser des questions en public (ce serait risqué mais, surtout, cela ne se fait pas: les journalistes n'aiment pas brûler leurs bonnes questions, qui les valorisent aux yeux de l'interviewé et les distinguent de leurs confrères), ils laisseront à table les places les plus proches des hôtes de marqué à leurs collègues seniors, ils prendront beaucoup de note (juste assez pour se relire aisément, pas trop pour éviter de passer pour des ignorants). Le même jeu 
se produit également, quoique plus rarement, du côté des chargés de R.P., lorsque ceux-ci se sont adjoints quelques stagiaires ou hôtesses d'appoint. On retrouvera ces néophytes à l'entrée des locaux, souhaitant modestement la bienvenue et présentant la farde de circonstances, souriant humblement et considérant avec respect manifeste les « grands professionnels des médias » qu'ils accueillent.

Ce constant effort d'auto-adaptation aux codes en vigueur s'expose évidemment aux erreurs de comportement et aux attitudes non pertinentes. C'est la une conséquence directe de l'extrême professionnalisation des rapports considérés, lesquels sont à la fois empreints de toute l'expérience accumulée et d'une rare qualité de civilité.

L'auteur de ces lignes en a personnellement fait la pénible expérience: invité, en compagnie d'une vingtaine d'autres journalistes beiges, dans un établissement thermal réputé de la côte normande, il crut bon de porter le complet veston dès son arrivée à l'hôtel (comportement que le grand luxe de ce dernier semblait recommander) alors que les confrères, tels des "clochards de palace", continuaient d'y évoluer en jean's et baskets. Il s'en est même fallu de peu pour que, ayant tiré la leçon pratique de cet épisode un peu ridicule, il ne se rende au « dîner officiel » dans cette tenue détendue, cependant que les collègues entre-temps, s'étaient enfin parés de leur cravate ou de leur robe de soirée...

Ces efforts constants de mutuel accommodement, ces réajustement successifs au cadre adéquat de l'interaction ne doivent toutefois pas faire penser à une quelconque spontanéité ou même à une sorte de bienveillance générale. Chaque acteur, professionnel des R.P. ou journaliste, invité ou témoin, et chacun à son rang, selon son rôle propre, sait exactement pourquoi il est la et agit de la sorte, pourquoi l'autre s'y trouve et réagit de manière complémentaire. $\mathrm{Si}$, en première approche, c'est sur le mode de la tromperie ${ }^{11}$ que semble se dérouler cette mise en scène fabriquée de toutes pièces par quelques artisans habiles des R.P., c'est en réalité en pleine conscience que les interlocuteurs tentent mutuellement de se séduire ou, à tout le moins, de gagner l'estime de l'autre. Bref, si mensonge il y a, c'est uniquement au regard d'un cadre déontologique qui n'est pas de mise ici. Nous parierons donc plus volontiers de pragmatisme, c'est-à-dire d'une tromperie sans réelle malice, une tromperie convenue qui, socialement et professionnellement, semble de bon aloi.

\section{La distinction comme corollaire de la ritualisation}

L'exposé qui vient d'être esquissé pourrait heurter un certain bon sens. Au fond, si la raison d'être de l'interaction n'est vraiment qu'une transmission d'information, pourquoi celle-ci ne se réduit - elle pas à un échange de cartons, de communiqués ou de dossier, en tout cas à une forme beaucoup plus rapide, sobre et assimilable qu'une "grosse opération » de relations publiques? Cette question, en apparence anodine, est peut-être la seule question qui vaille: pourquoi existe-t-il une pratique professionnelle de la communication? Ou, si l'on veut se faire plus précis : pourquoi faut-il que des médiateurs spécialisés interviennent la ou la quasi immédiateté serait peut-être possible et assurément moins coûteuse?

Plusieurs ordres de réponse sont possibles, du plus trivial (le corporatisme et la pression des milieux professionnels) au plus spécifique (une problématique d'image ne saurait se réduire à une transmission d'information). Tous sont a priori plausibles mais ils ne nous intéressent pas directement. Nous sommes en effet à la recherche d'une explication 
anthropologique qui nous permettrait d'assigner une motivation socio-culturelle à des comportements relevant quasiment, si l'on en croit une certaine littérature ${ }^{12}$ de la sphère technique.

L'hypothèse que nous avançons est simple : c'est la participation à un certain type de rituels qui meut les journalistes et les attachés de presse qui se rencontrent dans les circonstances qui nous intéressent ici. Non pas certes une participation peu consciente et presque fusionnelle mais, au contraire une adhésion aussi convenue et complice que nous avons pu le suggérer.

Le rituel, soit, mais... n'est-ce pas trop facile ? Les occasions de dépasser l'usage du terme "qui, dans la presse principalement, qualifie toute manifestation collective, un tant soi peu réglée ${ }^{13}$ ne sont pas si fréquentes, et voilé précisément qu'il s'en présente une. L'observateur qui participe a des rencontres de presse de l'ampleur de celles qu'il nous a été donné de suivre est frappé par l'abondance - et la richesse - des séquences répétitives d'actions (paroles, gestes, symboles...) dont la signification renvoie aux codes socioprofessionnels et culturels en vigueur dans le champ considéré. Ce sont donc bien la des rituels au sens où l'anthropologue l'entend généralement ${ }^{14}$.

31 Le développement des Communications de masse, en effet, a entraîné l'émergence d'un nouveau territoire, d'un « champ » spécifique au sens de Bourdieu, que l'on peut designer d'un mot comme étant celui où évoluent publiquement les médiateurs. Se constituant en système relativement autonome, ce champ a rapidement développé une autoorganisation sui generis, des codes spécifiques et, partant, des manifestations ritualisées. Il serait d'ailleurs utile d'en faire un inventaire exhaustif et minutieux, ainsi qu'une étude de type " génétique »: procédures de salutations et de congédiations, remises de présent et de petites gratifications, repas communs, accomplissements de représentations personnalisées, exposés, shows multimédias, échanges de questions-réponses, cocktails d'inauguration ou d'hommage... sont autant de cristallisations du travail des médiateurs, non pas spécifiques en eux-mêmes (on les retrouve dans d'autres secteurs de la vie sociale) mais agencés selon des codes particuliers pour les besoins de l'exercice professionnel de la communication.

32 Sans du tout entreprendre ici cet inventaire par ailleurs bien souhaitable, je signale incidemment, afin d'accentuer la continuité de ce qui se joue ici avec des pratiques culturelles plus anciennes, que ces manifestations participent au moins de deux registres distincts. D'une part, on rencontre de véritables " créations ", telle la conférence de presse, un rituel dont il me semble difficile de repérer l'équivalent dans des pratiques du passé. Mais l'essentiel des actions possibles constitue une réactivation, avec les moyens de la communication contemporaine, de pratiques ancestrales, telles certains usages de cours, des repas d'hôtes, les parties de chasse, etc ${ }^{15}$.

La fameuse définition de la culture par Goodenough, à savoir la culture entendue comme « tout ce qu'il faut savoir pour être membre $»^{16}$ s'applique ici à merveille. Le nec plus ultra de la prévisibilité surgissant lorsque, jointe aux(x) rituel(s), l'institutionnalisation des pratiques professionnelles vient fixer les séquences qui étaient en train de se cristalliser. C'est ainsi que, comme me le confiait dernièrement un chargé de R.P. appartenant àune des plus grandes agences au monde, «il devient de plus en plus difficile d'imaginer qu'une information d'entreprise puisse être transmise à la presse autrement que lors d'une conférence de presse - quelles qu'en soient les modalités. Toute tentative d'échapper à ce schéma traditionnel risque immédiatement d'être assimilée à un happening façon « Greenpeace ». 

professionnels de la presse et des R.P. réunis, n'en est pas moins terriblement contraignante : la pleine adhésion - d'attitudes - au rituel ou la soi-disant adhésion professée - au jeu social est indispensable. L'acteur qui s'en croirait dispensé serait immédiatement menacé de disqualification et ravalé au rang peu enviable d'outsider ayant indûment fait irruption dans le champ clos des médiateurs. Une catégorie d'intervenants en fait d'ailleurs l'expérience à ses dépens : celle des hauts responsables d'entreprises, souvent conviés au moment fort de la rencontre pour assurer un accueil flatteur, prononcer un discours ou « faire connaissance de la presse ». Leur fonction - et leur rang - les placent doublement en porte-à-faux : les journalistes ne s'adressent guère à eux que pour leur soumettre des points de technique ou de stratégie (mais jamais pour « entrer en relation » à l'aide, par exemple, d'un « Aimez-vous le tennis »), tandis que les chargés de R.P. endossent le mode hiérarchique que leur impose leur travail de commande. Sauf à être lui-même un charismatique des médias, le "patron", quelque peu égaré dans un univers qui n'est pas le sien, révélera donc que quelques-unes des clés du code lui échappent: tel viendra rejoindre les RP, qui saluent, sur la passerelle du bateau, les journalistes prenant congé, tel autre, assis dans l'inévitable position centrale qui lui est assignée au banquet, ne parviendra à converser ni avec les groupes de droite, ni avec ceux de gauche, tel autre enfin grand «classique » du genre - confondra le journaliste de la télévision publique avec un stagiaire venu pour un quotidien régional.

Notons que la contrainte et les vertus récupératrices du genre s'étendent très loin. J'ai ainsi pu assister au réinvestissement de la fonction, traditionnelle dans les milieux de cour, de fou de roi. L'animateur de radio qui endossa ce rôle le fit volontiers et en pleine conscience : le directeur de l'exploitation, commanditaire de l'opération, connaissait la réputation du personnage et l'avait invité sur le conseil de son chargé de RP. La situation étant fixée par ce mutuel et tacite consentement, le jeu a pu aller très loin, le «fou » se permettant de mettre le directeur en scène dans des blagues douteuses au cours du repas officiel. Tout le monde était ravi : le directeur, qui trouvait cela spirituel et " décapant " (sic), les journalistes, qui prenaient ainsi leur revanche sur les décideurs par l'entremise d'un des leurs, et le responsable des R.P. qui constatait que tout cela prenait décidément une tournure très favorable à ses objectifs de « bons contacts ».

Le caractère extrêmement lisse et poli, d'une part, et fortement ritualisé d'autre part, de ces grandes rencontres avec la presse explique en partie l'attitude, appréciée des journalistes, "des bons" chargés de R.P. Ceux-ci se gardent bien de "se mettre en avant ». Figures modernes d'Hermès, le discret messager des dieux qui en devient divinité à son tour, ces parfaits professionnels renforcent par leur attitude modeste la grandeur de ce qui se joue là. Reconnaissables au premier coup d'œil, ces personnages emblématiques du champ des médiateurs publics échappent au port convenu de la chemise rose et de la brillante cravate de soie, ne se sentent pas obligés de se jeter sur les journalistes lorsque ceux-ci arrivent, les laissent sans hésitation repartir sans carte de visite, vont plus volontiers jeter un œil en cuisine que s'enquérir de l'assiette de leur convive de gauche. Véritables « lieu-tenants » au sens noble du mot, ils confirment dans leur bonne tenue qu'ils ne sont la que pour pallier l'absence de «celui » qui donne à la réunion sa pleine signification. $\mathrm{Du}$ reste, pourquoi en rajouteraient-ils puisque, indubitablement, tout en eux indique qu'ils « en sont ».

voici ainsi parvenus devant la pierre de touche qui a guidé notre démarche: ces rituels, pour l'annoncer d'entrée de jeu, appartiennent nettement à la catégorie décrite 
par Pierre Bourdieu comme celles des « rites d'institution $»^{18}$. La force de ce petit texte du sociologue français reste intacte : reprochant aux « rites de passage » de Van Gennep leur caractère quasi tautologique, Bourdieu y recommande la seule question qui fasse progresser l'analyse, celle de la fonction sociale des rites. La réponse, lumineuse et universelle, qu'il donne à cette question complexe s'applique parfaitement dans le cas particulier qui nous occupe.

En première approche, nous l'avons dit, les pratiques d'un champ en voie de professionnalisation se sont peu à peu instituées elles-mêmes comme rites. Mais au-delà, elles tendent à ré-instituer les différences existantes, celles en l'occurrence qui séparaient déjà les différentes catégories de médiateurs entre eux mais que la célébration de l'appartenance commune au même champ contribuait à estomper. Les rituels ainsi conçus, en vertu de la belle formule latine réactualisée par Bourdieu, « enseignent la nage aux poissons $»^{19}$ : ils recadrent sans arrêt les acteurs au cœur de l'interaction et leur réapprennent inlassablement les us et coutumes liés a leur position. En reformulant, en représentant des différences préexistantes entre les inter-actants, ces rituels, que chacun renforce chaque fois qu'il les pratique, manifestent leur caractère impératif pour chacun. La situation, au fond, ne laisse pas d'être paradoxale: tous ces professionnels de la médiation sont en quelque manière égaux, puisqu'ils appartiennent au même champ professionnel, puisqu'ils partagent grosso modo la même formation, les mêmes relations. Mieux, ils sont pratiquement interchangeables dans leurs activités même car, surtout depuis la grande vogue des écoles de communication, il n'y a guère de doute que les journalistes pourraient valablement accomplir le travail des chargés de R.P, et vice versa ${ }^{20}$

Malgré cette identité quasi fusionnelle, c'est au contraire la différence, la distinction qui s'est ré instituée dans les manifestations que nous avons observées. Quelle(s) distinction (s) dira-t-on? Le tableau ci-après en donne une idée schématique. On notera que les deux axes majeurs de distinction socio-professionnelle, que j'ai appelés "prestige» et "vulnérabilité », induisent, en termes d'image de ces professionnels, des perceptions et des jugements tranchés : le journaliste jouit du prestige de la visibilité de sa profession, tandis que le chargé de R.P. pâtit de la relative discrétion où il s'installe. De manière symétrique, sur le second axe de cette structure en forme de chiasme, je relève que l'homme de presse souffre, par exemple, de sa "pauvreté ", tandis que le R.P. semble jouir des faveurs de l'argent, celui de son salaire (en Belgique, il est toujours notablement plus élevé que celui d'un journaliste) comme celui dont on fait crédit à l'entreprise qui l'emploie.

JOURNALISTES CHARGES DE R.P.

PRESTIGE

- de l'information «noble » - de l'entreprise représentée

- de l'indépendance - de la proximité des décideurs

- de la visibilité médiatique - de l'argent

VULNÉRABILITÉ

- des entreprises de presse - de la communication en général marchande

- du statut de « spectateurs » - de la dépendance stratégique

- du salaire peu élevé - de la discrétion de la fonction. 

de la communication. Ils le font doublement (les distinctions sont évidemment perçues avec acuité par les professionnels eux-mêmes mais aussi, de manière plus diffuse, par des agents extérieurs au système) et de façon durable, la multiplication des «communicateurs » et la stabilisation globale du nombre de journalistes ${ }^{21}$ ne pouvant qu'affermir ces clivages.

51 Nous affirmons donc, au terme d'une lecture socio-anthropologique de quelques expériences typiques, que les rites d'institution consacrés par les pratiques des professionnels de la communication dessinent nettement, à l'intérieur même du champ, deux configurations contrastées, la participation commune aux activités de médiation publique n'abolissant pas les distinctions persistantes qui opposent les acteurs entre eux. Elles les opposent certes sur un mode presque ludique (la représentation n'est jamais loin du jeu), trèsdoux (le «mensonge » professionnel n'est jamais manifesté comme tel), mais d'autant plus efficace que les clivages sont pris en relais par des codes et des rituels acceptés et célébrés par tous. par une firme pharmaceutique, il m'a été donné de vivre, presque sur le mode de la parabole, le mouvement même que notre réflexion a tenté d'esquisser.

Parti de la banlieue gantoise, d'un univers encore marqué par l'industrialisation, le bateau loué pour la circonstance a emmené la presse et le «staff» de la firme (entendez quatre chargés de R.P. attachés à la maison, deux hauts responsables, cinq chercheurs et quelques auxiliaires chargés de "l'intendance » de l'opération) dans un périple bucolique. Après le traditionnel café-croissant de bienvenue, nous nous sommes enfoncés dans le ventre du bateau pour y suivre la partie proprement informative. Les rideaux étaient tirés, mais les jours subsistant attestaient que nous étions assis tout juste au niveau de la rivière. Tandis que le bateau poursuivait sa marche en avant, tandis que les orateurs se succédaient devant l'écran du diaporama, plusieurs journalistes se tournaient avec regret vers les baies vitrées, déplorant de ne pouvoir admirer le paysage. D'abord prisonniers de l'information (leur métier !) c'est donc avec un certain soulagement qu'ils accueillirent les « officiants » (les deux plus jeunes attachés de R.P. du staff) venus ouvrir les rideaux à la fin de la brève séquence de questions-réponses. Presque aussitôt, nous regagnions le pont, où un barbecue parfait nous attendait. À cet instant s'accomplissait la fusion : tous égaux dans la file qu'il fallait suivre pour aller se servir, ensuite joyeusement mêlés - et serrés, exiguïté du lieu oblige - aux tables du repas, journalistes, responsables et chargés de R.P. se sont adonnés librement, sans plus de référence à l'information qui les réunissait, aux joies du repas en commun, la "communion », pourtant ne pouvait être éternelle; de fait, a peine de repas commencé, le bateau a fait demi-tour sur lui-même et a entrepris la descente vers Gand - prémice de fin prochaine - permettant enfin aux convives d'admirer le paysage qui leur avait été dérobé, a l'aller, au profit des exigences de l'information-prétexte (car tout ce qu'il fallait savoir, comme de coutume, se trouvait de toute façon dans les documents remis à chacun). Dans l'ambiance curieuse du repas qui s'achevait, les quartiers proches de Gand étaient rejoints. C'est le café - un autre passage obligé -, dégrisant les esprits et les corps, qui marqua définitivement le retour au point de départ : tous les journalistes débarquaient, le staff restait à bord. Même le responsable des R.P. qui avait accompagné le car de la presse de Bruxelles à Gand, restait pour l'heure avec les siens. Ite, missa est, retour a la distinction, chacun pouvait donc s'en retourner à son lieu et à son statut d'origine. 


\section{Relations de presse et relations publiques}

Si l'on veut donner à cette petite enquête une certaine portée générale (non seulement les relations de presse, mais les relations publiques comme rituels d'institution de distinction), il nous reste à indiquer en quel sens précisément, ces rencontres avec la presse sont des figures emblématiques des pratiques de relations publiques.

5 Ce point repose évidemment sur les acteurs eux-mêmes et les codes socio-culturels qu'ils partagent et actualisent, plutôt que sur le type d'organisation qu'ils entreprennent. On peut, ici aussi, miser sur l'institutionnalisation du secteur: si l'on accepte l'idée d'un champ professionnalisé de la communication, a l'intérieur même duquel s'organiserait une configuration encore plus déterminée, celles des relations publiques (au sens large), il n'y a pas de raison a priori de supposer que les acteurs se comporteraient d'une manière significativement différente de cette région du champ. J'indique ainsi - je ne démontre pas - que les autres pratiques de relations publiques seraient redevables du même style d'analyse que celle que nous venons de pratiquer ${ }^{23}$.

Au-delà d'un tel schéma interprétatif, le principe du pragmatisme professionnel et des distinctions institutionnalisées pourrait gagner de proche en proche d'autres discours « d'illustration et de défense de l'information pure » qui ne résisterait pas longtemps au regard socio-anthropologique.

7 Mais la relation (et donc la communication), au contraire de l'information, n'est pas susceptible d'être fausse ou exacte, mensongère ou sincère ${ }^{24}$. En tant que comportement intersubjectif, elle est aussi passible d'une analyse en termes de significations. Que la communication soit une figure du pragmatisme social n'en fait pas pour au tant un pur moyen, dépourvu de finalités relationnelles et éthiques. Indice d'un contexte relationnel déterminé par des impératifs professionnels, le "mensonge " dont il a été question renvoit ici au regard de celui qui le juge. Et la distinction, précisément, est dans le regard, non dans l'objet.

\section{NOTES}

1. Les écoles supérieures de communication comprennent généralement des départements ou des orientations relativement distinctes selon que l'on y enseigne le journalisme ou les relations publiques. Si cette distinction se justifie largement sur un plan pratique (les techniques professionnelles sont différentes), elle est en outre souvent légitimée par une distinction de droit entre Information et communication. C'est précisément contre cette dichotomie que nous nous insurgeons, à la lumière de nos observations sur le terrain.

2. Je fais évidemment allusion à une des propositions centrales de Watzlawick, Beavin et Jackson pour établir leur axiomatique de la communication: "Dans ce qui précède, noua avons fait allusion à un autre axiome en disant que toute communication suppose un engagement et définit par la suite la relation. C'est une autre manière de dire qu'une communication ne se borne pas à transmettre une Information, mais induit en même temps un comportement » (Watzlawick P, 
Beavin J.H., Jackson D. Une logique de la communication, trad. J. Morche, Paris, éd. du seuil, 1979, p. 49).

3. Heinich Nathalie, Relations publiques, relations en public, communication orale présentée à une journée d'études du Groupe de contact du F.NRS, consacré aux «Théories des relations publiques et théories de la communication ", le 18 mai 1992 (non publié).

4. Comme l'a bien fait remarquer Yves Winkin en empruntant cette distinction à Goffman, l'image globale de l'entreprise n'est pas seulement faite des éléments construits, maitrisés dans leur présentation publique (given), mais aussi de cette inévitable part de représentations immaîtrisées que l'entreprise ne peut s'empêcher de « lâcher » dans l'opinion (given off) Lire à ce sujet Winkin Yves, Publicité d'entreprise et maîtrise des impressions, in Reflets et perspectives de la vie économique, tome XXIX, $\mathrm{n}^{\circ} 2$ 1990. p. 152

5. Voir notre note $n^{\circ} 2$.

6. Jean Charron, Relations Between Journalists and Public Relations Practioners : Cooperation, Conflict and Negotiation, tiré des travaux du Groupe de Recherche sur les Journalistes et les Communicateurs (Université Laval), sous la direction de Florian SAUVAGEAU et Jacques LEMIEUX, 1991, T.A.P. à paraître.

7. Cet avis, à ranger parmi les propos auto-légitimateurs de la profession, m'était confié par un journaliste scientifique de la presse écrite qui avait déjà plus de quinze années d'expérience professionnelle. [Du reste, dans la suite de l'exposé, les citations placées entre guillemets et rapportées sans référence explicite renvoient chaque fois à des propos glanés sur le terrain]

8. En règle générale, la transmission de l'information-pré-texte est organisée dans la première partie de la rencontre et est immédiatement suivie du moment prévu pour répondre aux questions des journalistes. Cette phase de transmission est toujours "habillée " de manière convenue par les chargés de RP. (dossiers de presse contenus dans des fardées luxueuses, prospectus hauts en couleurs diffuses par 1a firme, le tout rangé dans des valisettes flatteuses, que le journaliste collectionne ou offre à ses enfants au terme du voyage...) et est de courte durée (une heure trente au maximum pour une conférence de presse classique, une matinée environ pour une séance de travail plus étoffée) Mais le meilleur reste encore à venir: déjeuner de travail, repas de fête, visite de l'entreprise, soirée culturelle,.... tout cela occupe évidemment bien plus de temps que la partie proprement informative.

9. Bourdieu Pierre, Esquisse d'une théorie de la pratique; Précédée de trois études d'ethnologie kabyle, Genève,librairie Droz.1972. p.183

10. Telle est, en substance, la définition du cadre primaire de l'expérience que donne Goffman dans sa " cadre-analyse » " GOFFMAN Erving, Les cadres de l'expérience, trad. d'Isaac Joseph, Paris éd. du Seuil, 1991, p. 30).

11. Le terme peut ici être admis au sens ordinaire ; par contre, il conviendrait moins bien avec la force que lui a donné Goffman en l'utilisant dans un sens précis, comme une des figures principales du registre de la fabrication (fabrication) Au contraire de la forme de duperie qu'implique généralement la fabrication (et plus particulièrement encore la tromperie), Il semble que nous soyons ici en présence d'une simple modalisation du cadre primaire : "Par mode (key), j'entends un ensemble de conventions par lequel une activité donnée, déjà pourvue d'un sens par l'application d'un cadre primaire, se transforme en une autre activité qui prend la première pour modèle mais que les participants considèrent comme sensiblement différente » (Goffman Erving, op, cit. p. 52). Toutefois, il n'est pas exclu que les règles déontologiques tacites en vigueur à l'Intérieur de ce champ soient vécues comme une tromperie au sens Goffmannien par des personnes extérieures au champ, et donc non averties. Ceci expliquerait peut-être la méfiance quasi viscérale dont les relations publiques font l'objet dans l'opinion publique.

12. j'ai déjà évoqué ce genre de discours prescriptif et techniciste en introduisant cette réflexion. Le malaise semble profond: même les grands ouvrages de référence des relations publiques semblent d'emblée marqués par cette dictature du «How to do?» (Cf. le Traité de relations 
publiques de C. Lougovoy et D. Huisman ou, plus ancien et marqué par les origines américaines des R.P, le célèbre Publics Relations d'E. Bernays) De sorte qu'il est légitime de se demander, avec Yves Winkin, «quelles théories pour quelles pratiques?» (Y. Winkin, Communication et relations publiques, quelles théories pour quelles pratiques? à paraître in Comnunication et relations publiques. B. Mangelinckx éd, Bruxelles, De Boeck).

13. Fabre Daniel, Le rite et ses raisons, in Terrain, $n^{\circ} 8$, avril 1987, p. 3.

14. Coupant court à une série d'équivoques subsistantes, l'ethno-sociologue Albert Platte a rappelé qu'il fallait considérer avec prudence l'association classique du rituel collectif et du lien social Synthétisant Les possibilités d'interpréter les rituels collectifs en échappant aux écueils de cette "épistémologie spontanée", il examine quelques pistes dont deux nous intéressent Spécialement. La première pose que le rituel implique un double processus: «la décontextualisation d'un message, d'un ensemble d'interactions sociales quotidiennes et la recontextualisation de ce même message et ces mêmes interactions sociales selon la logique propre du rituel. Cette formulation semble parfaitement convenir à l'utilisation spécifique qu'il en est fait dans le champ considéré de rituels sociaux existant par ailleurs. La seconde (la « cinquième hypothèse » dans le texte d'A Piette) situe l'efficacité maximale du rituel collectif en un point ou s'équilibrent « rappel spatio-temporel sécurisant » (Piette Albert, Lien social, rituel interstice, In Actes du XIII ${ }^{e}$ Colloque de l'A.I.S.L.F, tome Il. 1989, pp. 769 et 771) Cette dernière hypothèse, due à J-Scheff, pourrait peut-être expliquer, à y bien regarder, le subtil mélange de traditions et de créations originales qui s'instaure dans les manifestations de relations publiques considérées par les professionnels comme « les plus réussies » Mais l'examen détaillé de cette hypothèse dépasse le cadre du présent travail.

15. Yves Winkin, par exemple, a accompli ce genre de démarche socio-historique en découvrant des analogies aux pratiques "courtisanes » chères aux R.P dans les processus de curialisation décrits par Norbert Elias dans La société de cour (Cf. Winkin Yves, L'insaisissable statut théorique des relations publiques, in Guide des médias, G. Ringlet ed, Deurne, Kluwer, 1990).

16. Voici le passage décisif d'où cette belle définition est tirée: «Thus tested, the theory is a valid statement of what you have to know in order to operate as a member of the society and is, as such, a valid description of its culture " (Goodenough Ward, Cultural Anthropotogy and Linguistics, in Dell Hymes ed, Culture and Society, A Reader in Linguistics and Antropology, New York, Harper \& Row, 1964. p. 36).

17. «Prenons l'exemple des gestes. L'individu et le social s'y mêlent inextricablement; néanmoins, nous y sommes extrêmement sensibles, et nous y réagissons comme d'après un code secret et compliqué, écrit nulle part, connu de personne, entendu par tous [...] Comme toute conduite, le geste a des racines organiques mais les lois du geste, le code tacite des messages et des réponses transmis par le geste sont l'œuvre d'une tradition sociale complexe » (Sapir Edward. Anthropologie, trad. franc. Paris, éd de Minuit. 1967, p. 46). Comme le dit le grand anthropologue, les gestes ne sont qu'un exemple. Il ne faudrait pas faire beaucoup de violence à sa pensée, me semble-t-il, pour également appliquer cette thèse aux séquences répétitives de gestes, à savoir aux rituels.

18. Les rites d'institution est un texte repris comme deuxième chapitre de Ce que parler veut dire ; L'économie des échanges, linguistiques. Paris, Fayard, 1982, pp.121-134).

19. Bourdieu Pierre, loc. cit, p 123.

20. Les transferts sont fréquents entre les deux professions. En Belgique cependant. Ils ne s'opèrent guère que dans un sens (celui des journalistes qui deviennent chargés de relations publiques), le second métier étant bien mieux rémunéré que le premier.!

21. Il ne s'agit là, unefois encore, que de la situation en Belgique.

22. Petite rivière, au cours sinueux et champêtre, située au nord-ouest de la ville de Gand.

23. À condition que soit résolue la question épineuse du « répertoire » des pratiques qui font effectivement partie des relations publiques, ce qui supposerait un consensus sur la définition 
même des RP. A défaut de celui-ci, je signale d'emblée une pratique qui pose problème, celle du lobbying. Ou bien on la classe résolument du côté de la publicité (au sens litttéral), ou bien on y distingue, comme le font volontiers les professionnels, un lobbying « sain et démocratique », qui suppose une certaine transparence, et des pratiques "plus occultes", qui échappent à la déontologie des R-P. Dans les deux cas, le problème demeure de l'adéquation des notions générales aux réalités du terrain.

24. Cette remarque renvoie, une fois de plus, à l'axiomatique de Une logique de communication. (op. cit) et au noyau inévacuable de La communication ainsi décrite : le comportement. Comme tel, celui-ci n'est pas passable d'une analyse en termes de vérité ou de mensonge.

\section{RÉSUMÉS}

Les relations publiques en général, et les contacts de presse en particulier, constituent des phénomènes subtils de "relations en public" que seule l'observation participante permet d'observer et d'analyser dans toute leur épaisseur. Appliquant cette méthode à une série de contacts entre attachés de presse d'entreprises privées et Journalistes scientifiques, Alain Nivarlet montre que ces interactions sont régies par des codes spécifiques. professionnellement institués. On aperçoit ainsi les limites du rapprochement qui s'opère en ces circonstances entre journalistes et professionnels de la communication.

Public relations as a whole, and contacts with the press more particularly, are subtle phenomena of relations outside the private sphere that can only be analysed with the method of participant observation. Applying this ethnological enquiry to various contacts between public relations officers and sciences editors. Alain Nivarlet shows that these interactions are regulated by specific and professional instituted codes. This reveals the limits of the encounter in such circumstances between Journalists and communication's professionals.

\section{AUTEUR}

\section{ALAIN NIVARLET}

Alain Nivarlet est actuellement assistant en Arts et Sciences de la Communication (département Information et Médias) à l'Université de Liège (Belgique) et collaborateur régulier du Journal Le Soir (rubriques « Sciences » et « Médecine »). Sa thèse de doctorat, en cours d'élaboration, est une analyse anthropologique des mécanismes fondamentaux de la vulgarisation scientifique dans le domaine des sciences biomédicales. 